\section{PKA overexpression predicts treatment outcome in men with prostate cancer}

Protein kinase A type I (PKA) mediates cell processes such as DNA replication and cell proliferation in mammalian cells. Overexpression of PKA is associated with poor prognosis in studies of patients with breast, colon and lung cancers. Khor and colleagues investigated the predictive value of PKA overexpression in men with prostate cancer.

Both manual and image-analysis methods were used to score the immunohistochemical staining intensity of PKA expression in pretreatment biopsies obtained from 80 men who participated in the phase III, randomized, Radiation Therapy Oncology Group (RTOG) trial 86-10. This trial compared the effects of radiotherapy alone with radiotherapy plus short-term androgendeprivation therapy in men with prostate cancer.

On univariate analysis, significant associations were identified between PKA overexpression and cause-specific mortality $(P=0.037$ scored manually and $P=0.014$ scored by image analysis), distant metastasis $(P=0.029)$, local failure $P=0.011)$ and biochemical failure $(P=0.022)$. On multivariate analysis, the relationships between PKA overexpression and biochemical failure $(P=0.03)$, local failure $(P=0.002)$ and distant metastasis $(P=0.018)$ remained significant.

In this small group of men with prostate cancer, PKA overexpression seemed to be a stronger determinant of outcome after radiotherapy than the addition of short-term androgen-deprivation therapy. The authors suggest that PKA overexpression could be a potentially useful biomarker for patients with high-risk prostate cancer, but further studies are needed to confirm these results.

Original article Khor L-Y et al. (2008) Protein kinase A RI- $\alpha$ predicts for prostate cancer outcome: analysis of radiation therapy oncology group trial 86-10. Int J Radiat Oncol Biol Phys 71: 1309-1315

\section{Preoperative chemoradiotherapy improves outcomes in nonresectable rectal cancer}

Approximately $10-15 \%$ of primary rectal cancers are nonresectable, although preoperative radiotherapy can shrink these tumors and permit subsequent radical surgery to be performed. The randomized study by Brændengen and colleagues investigated whether the addition of chemotherapy to preoperative radiotherapy could improve downstaging, survival and recurrence rates in patients with nonresectable rectal cancer.

In total, 207 patients (aged $<75$ years) with primary, nonresectable or locally recurrent adenocarcinoma were randomly assigned to receive $50 \mathrm{~Gy}$ radiotherapy alone $(n=109)$ or 5 -fluorouracil concurrent with 50 Gy radiotherapy, followed by leucovorin $(n=98)$. Surgery was performed 5-8 weeks after the last radiation treatment.

At follow-up (median 61 months in survivors), $63 \%$ of patients who received combined therapy were treatment-failure-free compared with $44 \%$ in the radiotherapy group $(P=0.003)$. Cancer-specific survival at 5 years was significantly higher in the combination-therapy group ( $72 \%$ versus $55 \%$, $P=0.02)$. A trend towards improved overall survival in patients who received both therapies was evident, but the difference between the groups was not significant ( $66 \%$ versus $53 \%, P=0.09$ ). Local control rates were also higher in the combinationtherapy group. Grade 1 and 2 diarrhea was the most frequent acute toxicity. Patients who received combination therapy experienced significantly more grade 3 or 4 toxicity than those given radiotherapy alone $(28 \%$ versus $6 \%, P=0.001)$.

The authors conclude that preoperative chemoradiotherapy is well-tolerated, improves local control, time-to-treatment failure and cancerspecific survival compared with radiotherapy alone in patients with nonresectable rectal cancer.

Original article Brændengen M et al. (2008) Randomized phase III study comparing preoperative radiotherapy with chemoradiotherapy in nonresectable rectal cancer. J Clin Oncol 26: 3687-3694

\section{Fractionated total body irradiation and etoposide confer remission in $\mathbf{P h}^{+}$ALL}

For patients with Philadelphia-chromosomepositive acute lymphoblastic leukemia $\left(\mathrm{Ph}^{+} \mathrm{ALL}\right)$, allogeneic hematopoietic cell transplantation after induction chemotherapy is the optimal therapy. Laport and coauthors have reported outcomes of patients with $\mathrm{Ph}^{+} \mathrm{ALL}$ who received fractionated total body irradiation and high-dose etoposide.

The study included 79 patients with B-lineage ALL who underwent conditioning before hematopoietic cell transplantation from HLA-matched sibling donors. Participants received fractionated total body irradiation with either high-dose 